\title{
Annotations
}

\section{Seizures and steroids}

For over 40 years we have known that some children with otherwise intractable fits improve with steroids, ${ }^{1}$ and for over 30 years that a similar response may be obtained with adrenocorticotrophic hormone. ${ }^{2}$ Since that time a considerable amount of work has centred on infantile spasms, particularly on the relative efficacies of prednisone and adrenocorticotrophic hormone. For each report asserting the superiority of one, it is possible to find another giving the opposite view. Close comparison is confounded by the fact that drugs, dosage, and duration of treatment have varied between the trials. One recent paper ${ }^{3}$ was unable to find any convincing difference of effect between the two. Despite this, certain facts have emerged; more patients respond to adrenocorticotrophic hormone than to prednisone $;^{4}$ there are a few, however, who will respond to the latter but not the former. ${ }^{5}$ The relation between the spasms and hypsarrhythmia is not a close one as the electroencephalographic changes may appear considerably later than the onset of spasms. Recurrence of spasms after hormone treatment is rarely attended by reappearance of hypsarrhythmia and neither the spasms nor the hypsarrhythmia by themselves are likely to affect long term development. What conclusions can be drawn from all this work?

The first and most obvious conclusion is that adrenocorticotrophic hormone acts independently of its effect on the adrenal glands. It is just conceivable that adrenocorticotrophic hormone acts via steroid metabolites intermediate on the biosynthetic pathway; it is difficult otherwise to see how some patients do better on adrenocorticotrophic hormone and a few on prednisone. This also makes the clinical observation that pharmacological doses of adrenocorticotrophic hormone ( 80 IU) may be more effective than lower doses (20 IU), more plausible. Perhaps, most convincingly, adrenocorticotrophic hormone can control fits in adrenal suppressed patients, including those concurrently on prednisone, even in the presence of documented low serum cortisol concentrations. The most simple alternative is that adrenocorticotrophic hormone has a direct effect on neurons or glial cells, or both.

A second conclusion must be that infantile spasms are associated with almost every condition affecting infants known to the paediatric neurologists. It is unlikely that conditions as disparate as phenylketo- nuria, subdural haematomas, and tuberose sclerosis elicit this stereotypic response by precisely the same mechanisms. Once this heterogeneity of the condition is accepted, it is easier to see that more appropriate questions might be why some patients respond to one drug and not another, and does this tell us anything about what is actually going on? The nature of the disease process, particularly in the cryptogenic cases, raises as big a research challenge as when West described its devastating course 143 years ago.

Work has begun in this direction. Serotonin has been of interest since the observation that $50 \mathrm{H}$ tryptophan induced infantile spasms in infants with Downs syndrome. ${ }^{6}$ The same phenomenon can be reproduced in young guinea pigs, after which the spasms can be abolished entirely by steroids or by serotonin antagonists. Other myoclonic disorders in man including dyssinergia cerebellaris myoclonica (Ramsey Hunt syndrome), paramyoclonus multiplex, and L dopa induced myoclonus respond to the serotonergic blocking agent, methysergide. To expect that infantile spasms are simply caused by a central serotonin excess, however, underestimates the complexity of even the little we know of neurotransmitter circuitry. Indeed postanoxic myoclonus responds to $50 \mathrm{H}$ tryptophan. It may come as no surprise, therefore, that a recent study reported lower serotonin metabolite concentrations (5HIAA) in the cerebrospinal fluid of children with infantile spasms than in age matched controls. ${ }^{7}$ These conflicting pieces of evidence have been rather neatly reconciled by suggesting that the primary abnormality may lie in supersensitive serotonin receptors that consequently suppress presynaptic serotonin turnover and release. ${ }^{7}$ A similarly recent and perplexing observation is that although, as might be expected, children with infantile spasms have lower concentrations of the inhibitory neurotransmitter $\alpha$-aminobutyric acid in cerebrospinal fluid, the effect of adrenocorticotrophic hormone which abolished spasms was to reduce concentrations of $\alpha$-aminobutyric acid still further. ${ }^{8}$

It seems clear that we are unlikely to advance far from an empirical approach until we understand more about the site and mode of action of adrenocorticotrophic hormone in the brain. Confined not only to the anterior pituitary, it is also found along with beta endorphin in cell bodies in the hypothala- 
mus which project to the limbic system, thalamus, and brain stem. It is probable, therefore, that it acts as a neurotransmitter in its own right in specific subcortical circuits. It would be of considerable interest to know if there are functional connections between these cells and those of the pontomedullary raphe complex, which contains a high concentration of serotonergic neurons and which, it has been suggested, may modulate infantile spasms.

One only has to consider the gap between these speculations and our lack of understanding of the mechanism for ensuing mental handicap to see how many unanswered questions remain.

Another steroid responsive movement disorder in children is that of the opsoclonus myoclonus or 'dancing eyes' syndrome. This is frequently but not invariably a paraneoplastic effect associated with neuroblastoma. Whether the relation between the neuroblastoma and the brain rests upon a cross immunological basis, on an undescribed neurotransmitter secreted by the tumour, or some other mechanism is unknown, but once again this is a condition that responds dramatically to steroids.

Despite the increasing development of more effective anticonvulsants, it is worth remembering that older children with other types of seizure disorder may also respond to adrenocorticotrophic hormone in particular, or sometimes to steroids. ${ }^{5}$ Adrenocorticotrophic hormone may often be effective in children with somewhat delayed development who display a wide repertoire of seizure typesmyoclonus, akinetic, absence, complex, and major motor. It is in these children who have failed to respond adequately to a variety of anticonvulsant 'cocktails' that either adrenocorticotrophic hormone or a ketogenic diet should be considered. While each has its disadvantages, it should be borne in mind that apart from growth retardation, a Cushingoid face, and general obesity caused by the former treatment, more troublesome side effects such as hypertension and diabetes are most uncommon. It is sensible to keep an eye on electrolyte concentrations as severe hypokalaemia occasionally occurs. It is also good to know that children who are neurologically normal at the onset of seizures and who respond to adrenocorticotrophic hormone rarely relapse after a three month course.

It is, however, all too frequent that children who are already neurologically impaired and develop infantile spasms relapse on withdrawal of steroids. Since one is essentially buying time with a course of steroids in these patients, it may be argued that a more rational approach in the first instance is to attempt to control seizures with benzodiazepines or sodium valproate. The recommended dose of adrenocorticotrophic hormone varies between 20 and
$120 \mathrm{U} /$ day. I usually start with a dose of $40 \mathrm{U} /$ day and then increase it to $80 \mathrm{U} /$ day if after three to four weeks there has been no response. I then wait a further four weeks on this higher dose before concluding that this treatment is ineffective. If it is effective, I continue it for three to six months. If a child is normal initially and responds rapidly to adrenocorticotrophic hormone, I would opt for the shorter time. Prednisone is usually started at a dosage of $2 \mathrm{mg} / \mathrm{kg} / \mathrm{day}$, and in the event of response, this is reduced to a 10 to $20 \mathrm{mg}$ daily maintenance dose. Switching to alternate day steroids does not seem to result in loss of control. It must be realised that as none of these suggestions have been subjected to controlled clinical trials, they arise from rank empiricism.

Finally, however, one empirical claim deserves consideration. For some time there has been an anecdotal impression in the published reports that early, urgent adrenocorticotrophic hormone treatment of cryptogenic patients may preserve them from dementia. The key to this approach lies in the early recognition of infantile spasms for what they are. It is not always realised that before classic 'salaam' attacks develop, the fits may merely consist of a slight loss of postural tone, producing a momentary drooping of the trunk and dropping forward of the head. Seemingly innocuous, they rarely draw an urgent response from the parents, nor always from the general practitioner. Paediatricians have an important educational role in this situation.

\footnotetext{
References

${ }^{1}$ McQuarie I, Andersson JA, Ziegler MR. Observations on antagonistic effects of posterior pituitary and cortico-adrenal hormones in the epileptic subject. J Clin Endocrinol Metab 1942;2:406-10.

2 Klein R, Livingston S. The effect of adreno-corticotrophic hormone in epilepsy. $J$ Pediatr 1950;37:733-42.

${ }^{3}$ Hrachovy RA, Frost JP, Kellaway P, Zion TE. Double blind study of ACTH vs. prednisone therapy in infantile spasms. J Pediatr 1983;103:641-5.

4 Lacy JR, Penry JK. Infantile spasms. New York: Raven Press, 1976:79-105.

5 Snead OC, Benton JW, Myers GJ. ACTH and prednisone in childhood seizure disorders. Neurology (Minneap) 1983;33: 966-70.

- Coleman M. Infantile spasms associated with $50 \mathrm{H}$ tryptophan administration in patients with Down's syndrome. Neurology (Minneap) 1971;21:911-8.

7 Silverstein F, Johnston MV. Cerebrospinal fluid metabolites in patients with infantile spasms. Neurology (Minneap) 1984;34:102-5.

${ }^{8}$ Ito M, Kikawa H, Taniguchi T. Cerebrospinal fluid GABA levels in children with infantile spasms. Neurology (Minneap)
} 1984;34:235-8.

R O RoBinson Guy's Hospital, St Thomas's Street, London SE1 9RT 